\title{
A new geometrical perspective on Bohr-equivalence of exponential polynomials $^{1}$
}

\section{J.M. Sepulcre and T. Vidal}

Department of Mathematics, University of Alicante, 03080-Alicante, Spain.

ABSTRACT Based on Bohr's equivalence relation for general Dirichlet series, in this paper we connect the families of equivalent exponential polynomials with a geometrical point of view related to lines in crystal-like structures. In particular we characterize this equivalence relation, and give an alternative proof of Bochner's property referring to these functions, through this new geometrical perspective.

AMS Subject Classification: 30D20, 30Axx, 51Pxx, 51Lxx.

Key Words: Exponential polynomials, Functions of a complex variable, Crystal-like structure, Bohr's equivalence theorem, Bohr's equivalence relation, Exponential sums.

\section{Introduction}

In this paper we are going to handle the so-called exponential polynomials with real frequencies and complex constant coefficients, which are concrete subclasses of exponential sums of the form

$$
P_{1}(s) e^{\lambda_{1} s}+\ldots+P_{j}(s) e^{\lambda_{j} s}+\ldots, s \in \mathbb{C},
$$

where the $\lambda_{j}$ 's are complex numbers and the $P_{j}(s)$ 's are polynomials in $s$. More specifically, given $n \in \mathbb{N}$ and $\Lambda=\left\{\lambda_{1}, \ldots, \lambda_{n}\right\}$ an arbitrary finite set of distinct real numbers, we will consider the class of functions, say $\mathcal{P}_{\Lambda}$, consisting of exponential sums $P: \mathbb{C} \mapsto \mathbb{C}$ of the form

$$
P(s)=\sum_{j=1}^{n} a_{j} e^{\lambda_{j} s}, a_{j} \in \mathbb{C} \backslash\{0\}, \lambda_{j} \in \Lambda,
$$

where $\Lambda$ will be called a set of exponents or frequencies, and each $a_{j}$ will be called a coefficient of $P(s)$. In the extensive literature about the properties of exponential polynomials, we can find many interesting results and connections with other theories. In particular, the study of their zeros is a topic which appears in the first third of the twentieth century in relation with the development of differential equation theory (see for example [12]).

These classes of exponential polynomials represent a particular case of almostperiodic functions, which leads us to other applications in different areas of mathematics. The concept of almost periodicity was first studied by $\mathrm{H}$. Bohr and later generalized by V. Stepanov, H. Weyl and A.S. Besicovitch, among others. Under the topology of uniform convergence, given $\varepsilon>0$, Bohr's definition

\footnotetext{
${ }^{1}$ This is a preprint of an article published in Analysis and Mathematical Physics. The final authenticated version is available online at: https://doi.org/10.1007/s13324-021-00498-0
} 
involves the existence of a relatively dense set of vertical translates which differ less than $\varepsilon$ from the given function. In this context, S. Bochner interpreted the almost periodicity of a function in terms of the relative compactness of the family of its translates. So, in later literature, some authors defined almost periodic functions in this way (e.g. see $[2,4,5])$.

By analogy with Bohr's theory, we will consider in this paper an equivalence relation $\sim$ on the classes of exponential polynomials in the class $\mathcal{P}_{\Lambda}$ (see Definition 18 and compare with [9, Definition 3]) which is certainly based on that of Bohr concerning general Dirichlet series (for which the set of exponents forms a strictly increasing sequence of positive numbers tending to infinity). Bohr used it in that case in order to get so-called Bohr's equivalence theorem, which shows that equivalent Dirichlet series take the same values in certain sets in the complex plane (e.g. see $[1,11]$ and the recent paper [10]).

On the other hand, from a physical point of view, a crystal is a solid where the atoms (molecules or ions) constitute a periodic arrangement, forming a crystal lattice that extends in all directions. Moreover, a quasicrystal consists of arrays of atoms that are ordered but not strictly periodic. In this sense, various notions of almost periodicity were studied for example in [7] in connection with quasicrystals.

In connection with this physical point of view, in the first part of this paper we will follow the ideas involved in a crystal-like structure. In fact, given a grid pattern in $\mathbb{R}^{n}$ generated by repeating unit cells, we will consider a segmentation process of a prefixed line in $\mathbb{R}^{n}$, identified by a point $\mathbf{v}$ and a vector $\boldsymbol{\lambda}$, from the reduction to its equivalent modulus in the origin cell (see Definition 2). This new set will be denoted as $L_{\mathbf{v}, \boldsymbol{\lambda}}$ and we will prove that its closure provides a finite set of affine subspaces (see Proposition 9). Furthermore, we will analyze the strong connection between the sets $L_{\mathbf{v}, \boldsymbol{\lambda}}$ and $L_{\mathbf{w}, \boldsymbol{\lambda}}$ when $\mathbf{w}$ is chosen in the closure of $L_{\mathbf{v}, \boldsymbol{\lambda}}$ (see Proposition 13).

In Section 4, we will present some functions which, by evaluating them on certain lines in $\mathbb{R}^{n}$, generate the images of exponential polynomials in the classes $\mathcal{P}_{\Lambda}$. We will see that these generating functions are periodic in each of its coordinates, which leads to an interesting perspective which is related to that of crystal structures. Through this geometrical point of view, i.e. in terms of the associated sets $L_{\mathbf{v}, \boldsymbol{\lambda}}$, we will characterize in Section 5 the equivalence relation $\sim$ on the class $\mathcal{P}_{\Lambda}$ (see Theorem 24 ), and we will give an alternative proof of Bohr's equivalence theorem restricted to the classes of exponential polynomials in $\mathcal{P}_{\Lambda}$ (see Proposition 21).

In the last section, we will use this geometric approach to give an alternative proof of the fact that, with the norm of the uniform convergence, every class of equivalent exponential polynomials is compact (see Proposition 27, which is a particular case of [9, Proposition 2] improving Bochner's property). This approach was a central driver behind the development of [9, Proposition 2], but it is explicitly shown in this paper. As a consequence of it, on the one hand, it will be shown that every sequence of the set of vertical translates of an exponential polynomial has a subsequence that converges uniformly to an equivalent exponential polynomial (see Corollary 28 and compare with [9, Theorem 4]) and, 
on the other hand, we will prove that, given two arbitrary equivalent exponential polynomials, one of them can be approximated by vertical traslations of the other (see Corollary 29 and compare with [9, Corollary 5]). Moreover, the results of this last section can be easily extended to other topologies.

\section{Segmentation process of a line}

In this preliminary part, which is provided here as a means for the reader to readily justify the subsequent assertions, we will consider a crystal grid pattern which is generated in the Euclidean space $\mathbb{R}^{n}$ from repeating origin cells.

Definition 1 Given $n \in \mathbb{N}$, let $M=\left\{m_{1}, \ldots, m_{n}\right\}$ be an arbitrary set of $n$ integer numbers and let $C_{m_{1}, \ldots, m_{n}} \subset \mathbb{R}^{n}$ denote the Cartesian product

$$
\left[2 m_{1} \pi, 2\left(m_{1}+1\right) \pi\right) \times \cdots \times\left[2 m_{n} \pi, 2\left(m_{n}+1\right) \pi\right) .
$$

We will say that each $C_{m_{1}, \ldots, m_{n}}$ is a cell in $\mathbb{R}^{n}$, or simply cell. Moreover, if $m_{1}=\ldots=m_{n}=0$, then $C=C_{0, \ldots, 0}$ will be called the origin cell in $\mathbb{R}^{n}$, or simply origin cell.

Given $n \in \mathbb{N}$, note that the sides of each cell have length $2 \pi$ and the set of all the cells in $\mathbb{R}^{n}$ generates a grid pattern in $\mathbb{R}^{n}$. Moreover, if we take

$$
\mathbf{p}_{m_{1}, \ldots, m_{n}}=\left(2 m_{1} \pi, \ldots, 2 m_{n} \pi\right), m_{j} \in \mathbb{Z},
$$

then each cell $C_{m_{1}, \ldots, m_{n}}$ is of the form

$$
C_{m_{1}, \ldots, m_{n}}=C_{0, \ldots, 0}+\mathbf{p}_{m_{1}, \ldots, m_{n}} .
$$

As we will show later, it is worth noting that $C_{0, \ldots, 0}$ is the basic cell which we have chosen to implement a certain segmentation process. Specifically, given a line defined by a point $\mathbf{v}$ and a vector $\boldsymbol{\lambda}$ in $\mathbb{R}^{n}$, we will use the reduction map taking each element of $\mathbb{R}^{n}$ to its equivalent modulus in the period $[0,2 \pi)$. In this sense, the set $L_{\mathbf{v}, \boldsymbol{\lambda}}$ that we next define is obtained from this process and it will play a very important role in our paper.

Definition 2 Given $n \in \mathbb{N}$, consider a line $A_{\mathbf{v}, \boldsymbol{\lambda}}$ in $\mathbb{R}^{n}$ given by $\left\{\mathbf{x} \in \mathbb{R}^{n}\right.$ : $\mathbf{x}=\mathbf{v}+t \boldsymbol{\lambda}, t \in \mathbb{R}\}$, where $\mathbf{v} \in \mathbb{R}^{n}$ is a point of the line and $\boldsymbol{\lambda}=\left(\lambda_{1}, \ldots, \lambda_{n}\right)$ is a vector parallel to the line. Let $\left.R: \mathbb{R}^{n} \mapsto[0,2 \pi) \times \ldots n\right) \times[0,2 \pi)$ be the reduction map taking each element $\left(y_{1}, \ldots, y_{n}\right) \in \mathbb{R}^{n}$ to its equivalent modulus in the period $[0,2 \pi)$. The set of points $L_{\mathbf{v}, \boldsymbol{\lambda}}=R\left(A_{\mathbf{v}, \boldsymbol{\lambda}}\right)$, which is in the origin cell of $\mathbb{R}^{n}$, will be called the segmentation of the line $A_{\mathbf{v}, \boldsymbol{\lambda}}$ or the reduction of the line $A_{\mathbf{v}, \boldsymbol{\lambda}}$ to its equivalent modulus in the origin cell.

Equivalently, $L_{\mathbf{v}, \boldsymbol{\lambda}}$ can be defined as the set of points in the origin cell $C=[0,2 \pi) \times \ldots n) \times[0,2 \pi)$ of the type:

$$
\begin{aligned}
& L_{\mathbf{v}, \boldsymbol{\lambda}}=\left\{\mathbf{x} \in C: \mathbf{x}=\mathbf{p}_{m_{1}, \ldots, m_{n}}+\mathbf{v}+t \boldsymbol{\lambda}\right. \\
& \left.\qquad \text { for some } \mathbf{p}_{m_{1}, \ldots, m_{n}} \text { of form (2.1) and } t \in \mathbb{R}\right\} .
\end{aligned}
$$


Remark 3 Note that, without loss of generality, the point $\mathbf{v}=\left(v_{1}, v_{2}, \ldots, v_{n}\right)$ can be chosen so that each $v_{k} \in[0,2 \pi)$. In fact, it is clear that $L_{\mathbf{v}, \boldsymbol{\lambda}}=L_{\mathbf{v}+\mathbf{p}, \boldsymbol{\lambda}}$, with $\mathbf{p}=\left(2 m_{1} \pi, 2 m_{2} \pi, \ldots, 2 m_{n} \pi\right), m_{k} \in \mathbb{Z}$, for each $k=1, \ldots, n$. Moreover, if we take a point $\mathbf{w} \in L_{\mathbf{v}, \boldsymbol{\lambda}}$, then $L_{\mathbf{v}, \boldsymbol{\lambda}}=L_{\mathbf{w}, \boldsymbol{\lambda}}$.

Example 4 Consider $n=2, \lambda_{1}=\log 2, \lambda_{2}=\log 3$ and $v_{1}=v_{2}=0$. Then $A_{\mathbf{v}, \boldsymbol{\lambda}}$ is the line given by $y=\frac{\log 3}{\log 2} x$ and an approximation to the set $L_{\mathbf{v}, \boldsymbol{\lambda}}=R\left(A_{\mathbf{v}, \boldsymbol{\lambda}}\right)$ is represented in Figure 1.
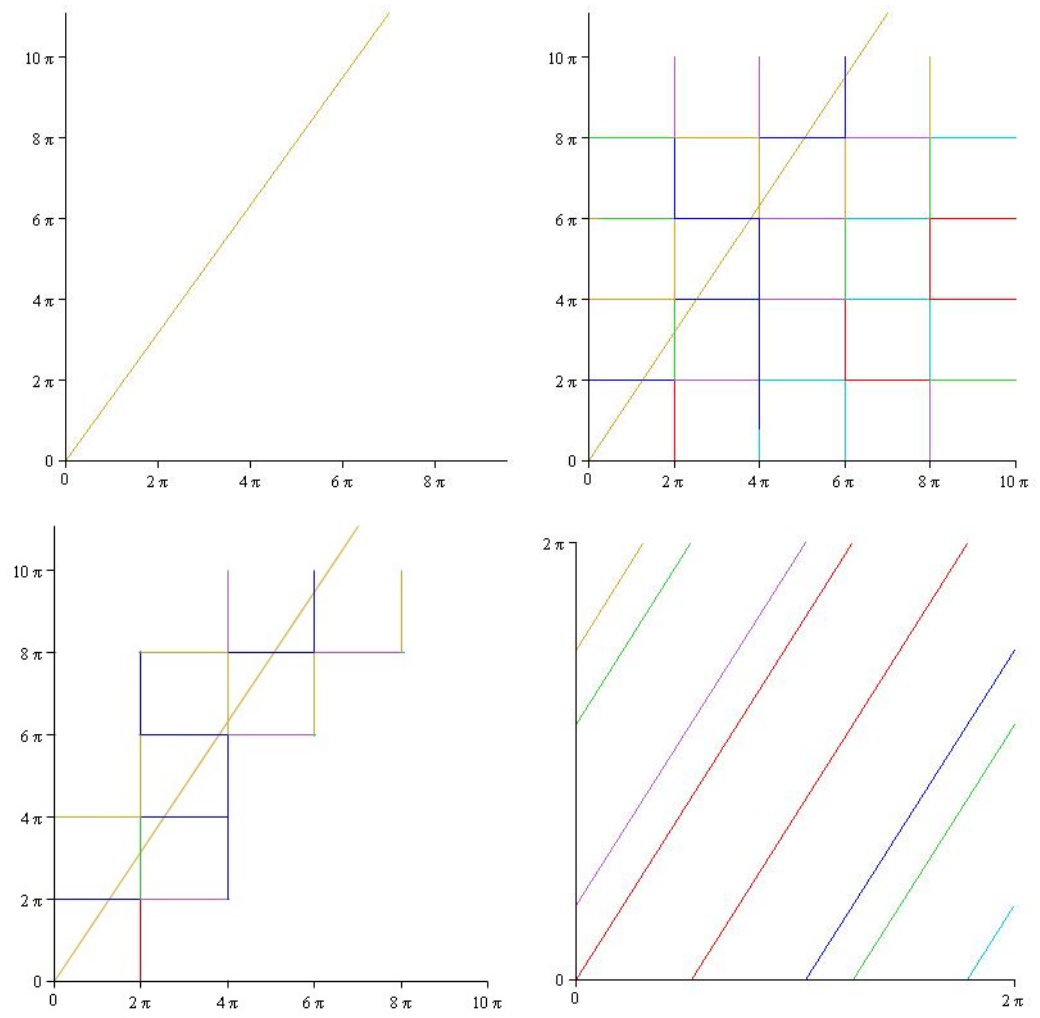

Figure 1: Representation of the set $A_{\mathbf{v}, \boldsymbol{\lambda}}$, the cells $C_{m_{1}, m_{2}}$ and the set $L_{\mathbf{v}, \boldsymbol{\lambda}}$ of Example 4.

Remark 5 Definitions 1 and 2 can be generalized in the following way. Fixed $n \in \mathbb{N}, c>0$ and a line $A_{\mathbf{v}, \boldsymbol{\lambda}}=\left\{\mathbf{x} \in \mathbb{R}^{n}: \mathbf{x}=\mathbf{v}+t \boldsymbol{\lambda}, t \in \mathbb{R}\right\} \subset \mathbb{R}^{n}$, we can consider $\left.R_{c}: \mathbb{R}^{n} \mapsto\left[0, \frac{2 \pi}{c}\right) \times \cdots n\right) \times\left[0, \frac{2 \pi}{c}\right)$ the reduction map taking each element $\left(y_{1}, \ldots, y_{n}\right) \in \mathbb{R}^{n}$ to its equivalent modulus in the period $\left[0, \frac{2 \pi}{c}\right)$. Then the set of points $L_{\mathbf{v}, \boldsymbol{\lambda}}^{c}=R_{c}\left(A_{\mathbf{v}, \boldsymbol{\lambda}}\right)$ will be called the reduction of the line $A_{\mathbf{v}, \boldsymbol{\lambda}}$ to its equivalent modulus in the origin cell of length $\frac{2 \pi}{c}$. Equivalently, if we take

$$
\mathbf{p}_{c, m_{1}, \ldots, m_{n}}=\left(\frac{2 m_{1} \pi}{c}, \ldots, \frac{2 m_{n} \pi}{c}\right), m_{j} \in \mathbb{Z}
$$


then

$$
\begin{aligned}
L_{\mathbf{v}, \boldsymbol{\lambda}}^{c}:=\left\{\mathbf{x} \in\left[0, \frac{2 \pi}{c}\right) \times \cdots^{n)} \times\left[0, \frac{2 \pi}{c}\right): \mathbf{x}=\mathbf{p}_{c, m_{1}, \ldots, m_{n}}+\mathbf{v}+\frac{t}{c} \boldsymbol{\lambda}\right. \\
\left.\qquad \text { for some } \mathbf{p}_{c, m_{1}, \ldots, m_{n}} \text { of the form (2.2) and } t \in \mathbb{R}\right\} .
\end{aligned}
$$

Moreover, the image of the sets $L_{\mathbf{v}, \boldsymbol{\lambda}}^{c}$ are invariant except for the scale factor (see for example Figure 2).
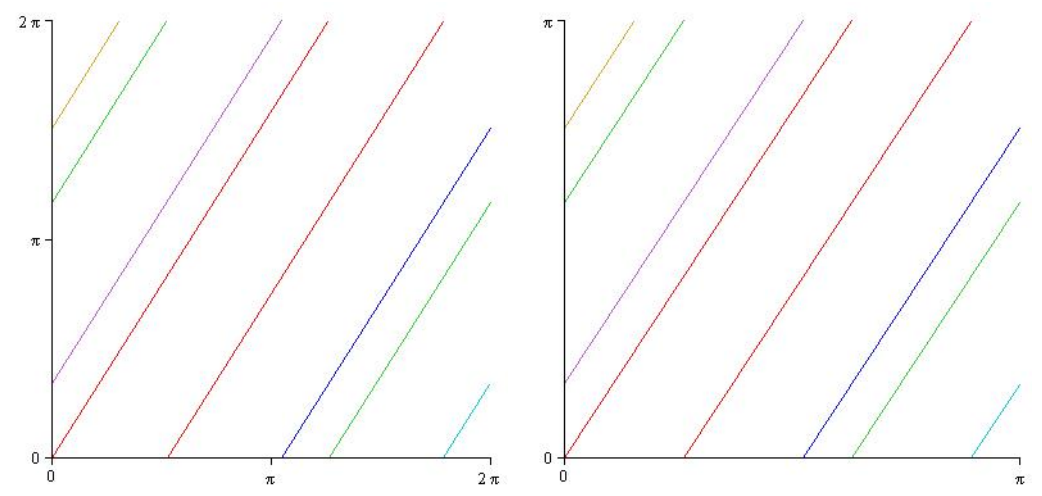

Figure 2: The representation of the set $L_{\mathbf{v}, \boldsymbol{\lambda}}^{2 \pi}$ in comparison with the set $L_{\mathbf{v}, \boldsymbol{\lambda}}^{\pi}$, with $\mathbf{v}=(0,0)$ and $\boldsymbol{\lambda}=(\log 2, \log 3)$.

\section{The density of the sets $L_{\mathrm{v}, \lambda}$}

We next study the closure of the sets $L_{\mathbf{v}, \boldsymbol{\lambda}}$ of Definition 2 associated with a line $A_{\mathbf{v}, \boldsymbol{\lambda}}$, with $\boldsymbol{\lambda}=\left(\lambda_{1}, \ldots, \lambda_{n}\right)$. Under $\mathbb{Q}$-linear independence of the set $\left\{\lambda_{1}, \ldots, \lambda_{n}\right\}$, we first prove that the set $L_{\mathbf{v}, \boldsymbol{\lambda}}$ almost fills up the closure of the origin cell for any point $\mathbf{v}$ in $\mathbb{R}^{n}$, which means that the origin cell is densified by the set $L_{\mathbf{v}, \boldsymbol{\lambda}}$ which is a union of parallel segments.

Proposition 6 Let $\left\{\lambda_{1}, \ldots, \lambda_{n}\right\}$ be a set of $n \geq 1$ real numbers which are linearly independent over the rationals, and let $\mathbf{v} \in \mathbb{R}^{n}$. Then

$$
\overline{L_{\mathbf{v}, \boldsymbol{\lambda}}}=[0,2 \pi] \times \cdots^{n)} \times[0,2 \pi],
$$

where $\boldsymbol{\lambda}=\left(\lambda_{1}, \ldots, \lambda_{n}\right)$.

Proof. Given $n \in \mathbb{N}$, consider $\boldsymbol{\lambda}=\left(\lambda_{1}, \ldots, \lambda_{n}\right)$ and $\mathbf{v} \in \mathbb{R}^{n}$. Take $\varepsilon>0$ and $\left.\left(d_{1}, \ldots, d_{n}\right) \in(0,2 \pi) \times \ldots n\right) \times(0,2 \pi)$. We will show the existence of a point $\left(x_{1}, \ldots, x_{n}\right) \in L_{\mathbf{v}, \boldsymbol{\lambda}}$ such that $\left|x_{l}-d_{l}\right|<\varepsilon$ for each $l=1, \ldots, n$. Since the elements of $\left\{\lambda_{1}, \ldots, \lambda_{n}\right\}$ are linearly independent over the rationals, by Kronecker's theorem [6, p.382], there exist a real number $t$ and integer numbers $m_{1}, \ldots, m_{n}$ such that

$$
\left|t \frac{\lambda_{l}}{2 \pi}-m_{l}-\frac{d_{l}-v_{l}}{2 \pi}\right|<\varepsilon \text { for each } l=1, \ldots, n,
$$


which means that

$$
v_{l}+t \lambda_{l}-2 m_{l} \pi=d_{l}+\eta_{l}, l=1, \ldots, n
$$

for some $\eta_{l}$ such that $\left|\eta_{l}\right|<\varepsilon$. Hence $\left|x_{l}-d_{l}\right|<\varepsilon$, with $x_{l}:=v_{l}+t \lambda_{l}-2 m_{l} \pi$. Note that, by choosing $\varepsilon$ sufficiently small $\left(0<\varepsilon<\min \left\{d_{l}, 2 \pi-d_{l}: l=1, \ldots, n\right\}\right)$, we get $x_{l} \in[0,2 \pi)$ for each $l=1, \ldots, n$. Hence $\mathbf{x}=\left(x_{1}, \ldots, x_{n}\right) \in L_{\mathbf{v}, \boldsymbol{\lambda}} \subset C$. Consequently, we have proved that $\left.(0,2 \pi) \times \ldots^{n}\right) \times(0,2 \pi) \subset \overline{L_{\mathbf{v}, \boldsymbol{\lambda}}}$ and, by taking into account $\overline{L_{\mathbf{v}, \boldsymbol{\lambda}}} \subset C$, we conclude that

$$
\overline{L_{\mathbf{v}, \boldsymbol{\lambda}}}=[0,2 \pi] \times \cdots^{n)} \times[0,2 \pi] .
$$

Remark 7 Regarding the proposition above, other stronger results such as Kronecker-Weyl theorem (see for example [8, Appendix 8, Theorem 1]) can be used in order to obtain the Lebesgue measure of $L_{\mathbf{v}, \boldsymbol{\lambda}}$.

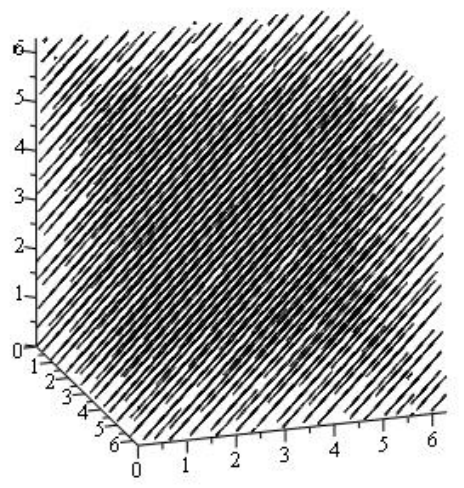

Figure 3: Approximation to the set $L_{\mathbf{0}, \boldsymbol{\lambda}}$ with $\boldsymbol{\lambda}=(\log 2, \log 3, \log 5)$.

Example 8 Let $\boldsymbol{\lambda}=(\log 2, \log 3, \log 5)$. Proposition 6 shows that

$$
\overline{L_{\mathbf{v}, \boldsymbol{\lambda}}}=[0,2 \pi] \times[0,2 \pi] \times[0,2 \pi], \text { for any } \mathbf{v} \in \mathbb{R}^{3} .
$$

See also Figure 3, which presents an approximation to this set. An equivalent image is obtained by choosing an arbitrary vector $\boldsymbol{\lambda}$ whose components are linearly independent over the rational numbers, but with the difference consisting of that the segments are aligned with this new chosen vector.

Without the hypothesis of the linear independence over $\mathbb{Q}$ of the elements $\left\{\lambda_{1}, \ldots, \lambda_{n}\right\}$, Proposition 6 can be generalized in the following way. Since the case $n=1$ is plain, from now on we will consider $n \geq 2$. 
Proposition 9 Given a point $\mathbf{v} \in \mathbb{R}^{n}$ and a vector $\boldsymbol{\lambda}=\left(\lambda_{1}, \ldots, \lambda_{n}\right)$, let $k$ be the dimension of the $\mathbb{Q}$-vector space generated by $\left\{\lambda_{1}, \ldots, \lambda_{n}\right\}$. Then there exists $m \in \mathbb{N}$ such that

$$
\overline{L_{\mathbf{v}, \boldsymbol{\lambda}}}=\left(\bigcup_{j=1}^{m} A_{j}\right) \cap\left([0,2 \pi] \times \ldots^{n)} \times[0,2 \pi]\right),
$$

for some $k$-dimensional affine subspaces $A_{j}$ in $\mathbb{R}^{n}, j=1, \ldots, m$.

Proof. Let $k$ be the dimension of the $\mathbb{Q}$-vector space generated by $\left\{\lambda_{1}, \ldots, \lambda_{n}\right\}$. Consider $\{1, \ldots, n\}=I_{1} \cup I_{2}$, with $I_{1} \cap I_{2}=\emptyset$ and $\sharp I_{1}=k$, such that the elements in $\left\{\lambda_{j}: j \in I_{1}\right\}$ are linearly independent over the rational numbers and

$$
\lambda_{j}=\sum_{l \in I_{1}} r_{j, l} \lambda_{l}, j \in I_{2},
$$

for some $r_{j, l} \in \mathbb{Q}, j \in I_{2}, l \in I_{1}$. Take $r_{j, l}=\frac{p_{j, l}}{q_{j, l}}$ with $p_{j, l}, q_{j, l} \in \mathbb{Z}$ such that $\operatorname{gcd}\left\{p_{j, l}, q_{j, l}\right\}=1$ if $p_{j, l} \neq 0$, and take $q_{j, l}=1$ if $p_{j, l}=0$. Also, let $q_{j}$ denote $\operatorname{lcm}\left\{q_{j, l}: l \in I_{1}\right\}$ for each $j \in I_{2}$. Fixed $\mathbf{v}=\left(v_{1}, v_{2}, \ldots, v_{n}\right) \in \mathbb{R}^{n}$, note that if $\mathbf{x}=\mathbf{v}+t \boldsymbol{\lambda}$, with $t \in \mathbb{R}$, it is clear that

$$
x_{j}=\left(v_{j}-\sum_{l \in I_{1}} r_{j, l} v_{l}\right)+\sum_{l \in I_{1}} r_{j, l} x_{l}, j \in I_{2},
$$

which provide $d$-dimensional affine subspaces in $\mathbb{R}^{k+1}$, for some $d \leq k$. That means that the points of the form $\mathbf{x}=\mathbf{v}+t \boldsymbol{\lambda}, t \in \mathbb{R}$, belong to these affine subspaces. Now, since the points of $L_{\mathbf{v}, \boldsymbol{\lambda}} \subset C$ are of the form given by $\mathbf{x}=\left(2 m_{1} \pi, \ldots, 2 m_{n} \pi\right)+\mathbf{v}+t \boldsymbol{\lambda}$ for some $t \in \mathbb{R}$ and $m_{j} \in \mathbb{Z}$, each equation (3.1) generates a finite amount of parallel affine subspaces in $\mathbb{R}^{n}$, say $A_{j, 1}, \ldots, A_{j, i_{j}}$, such that $L_{\mathbf{v}, \boldsymbol{\lambda}} \subset \bigcup_{l=1}^{i_{j}} A_{j, l}$. Indeed, for each $l \in I_{1}$ take $x_{l}=v_{l}+2 \pi e_{l} q$, where $e_{l} \in \mathbb{Z}$ and $q:=\operatorname{lcm}\left\{q_{j}: j \in I_{2}\right\}$. Then, by (3.1), we have for each $j \in I_{2}$ that $x_{j}=v_{j}+2 \pi d_{j}$ for some $d_{j} \in \mathbb{Z}$, and consequently $R\left(x_{1}, \ldots, x_{n}\right)=R\left(v_{1}, \ldots, v_{n}\right)$, where $R$ is the reduction map taking each element $\left(y_{1}, \ldots, y_{n}\right) \in \mathbb{R}^{n}$ to its equivalent modulus in the period $[0,2 \pi)$. Also, since $\left\{\lambda_{j}: j \in I_{1}\right\}$ is linearly independent over the rationals, Proposition 6 gives

$$
\overline{L_{\mathbf{v}^{*}, \boldsymbol{\lambda}^{*}}}=[0,2 \pi] \times \cdots^{k)} \times[0,2 \pi],
$$

where $\boldsymbol{\lambda}^{*}$ and $\mathbf{v}^{*}$ are the subvectors of $\boldsymbol{\lambda}$ and $\mathbf{v}$, respectively, determined by the components $I_{1}$. That is, the components $x_{l}, l \in I_{1}$, of (3.1) go through almost all the values and thus the affine subspaces provided by (3.1) are $k$-dimensional and they are almost filled up in the origin cell. Hence

$$
\overline{L_{\mathbf{v}, \boldsymbol{\lambda}}}=\left(\bigcup_{j \in I_{2}}\left(\bigcup_{l=1}^{i_{j}} A_{j, l}\right)\right) \cap\left([0,2 \pi] \times \ldots^{n)} \times[0,2 \pi]\right) .
$$


Remark 10 Under the same conditions as Proposition 9, let $V$ be the $\mathbb{Q}$-vector space generated by $\Lambda=\left\{\lambda_{1}, \ldots, \lambda_{n}\right\}$. If $\Lambda=\Lambda_{1} \cup \Lambda_{2}$, with $\Lambda_{1} \cap \Lambda_{2}=\emptyset$ and $\sharp \Lambda_{1}=k=\operatorname{dim}(V)$, then the affine subspaces $A_{j}$ of Proposition 9 are uniquely determined by the rational coefficients so that the elements in $\Lambda_{2}$ can be expressed as a $\mathbb{Q}$-linear combination of the elements of $\Lambda_{1}$ and their position with respect to the elements of this set. In fact, this is clear from equations (3.1) which determine $A_{j}$. Moreover, it is plain that the sets $\overline{L_{\mathbf{v}, \boldsymbol{\lambda}}}$ and $\overline{L_{\mathbf{w}, \boldsymbol{\lambda}}}$ are formed from parallel affine subspaces for any $\mathbf{v}$ and $\mathbf{w}$ in $\mathbb{R}^{n}$.

Remark 11 Propositions 6 and 9 can be easily generalized to the sets $L_{\mathbf{v}, \boldsymbol{\lambda}}^{c}$, with a prefixed $c>0$, defined in Remark 5. In this case, the cells have length $\frac{2 \pi}{c}$.

The following results concern the strong connection between the sets $L_{\mathbf{v}, \boldsymbol{\lambda}}$ and $L_{\mathbf{w}, \boldsymbol{\lambda}}$ when $\mathbf{w}$ is chosen in the closure of $L_{\mathbf{v}, \boldsymbol{\lambda}}$. As we will see later, this connection will give rise to a certain characterization of the equivalence of exponential polynomials with the same sets of exponents.

Lemma 12 Given a point $\mathbf{v} \in \mathbb{R}^{n}$ and a vector $\boldsymbol{\lambda}=\left(\lambda_{1}, \ldots, \lambda_{n}\right)$, consider $\mathbf{w} \in \overline{L_{\mathbf{v}, \boldsymbol{\lambda}}}$. Then $L_{\mathbf{w}, \boldsymbol{\lambda}} \subset \overline{L_{\mathbf{v}, \boldsymbol{\lambda}}}$.

Proof. Given $\mathbf{w} \in \overline{L_{\mathbf{v}, \boldsymbol{\lambda}}}$, by reductio ad absurdum suppose the existence of $\mathbf{x} \in L_{\mathbf{w}, \boldsymbol{\lambda}}$ satisfying $\mathbf{x} \notin \overline{L_{\mathbf{v}, \boldsymbol{\lambda}}}$. Without loss of generality, suppose $x_{i} \neq 0$ for each $j=1, \ldots, n$ (recall that $\overline{L_{\mathbf{v}, \boldsymbol{\lambda}}}$ is formed from affine subspaces). Under these conditions, on the one hand it is accomplished that $\mathbf{x}=\mathbf{w}+t_{0} \boldsymbol{\lambda}+\mathbf{p}$, for some $t_{0} \in \mathbb{R}$ and $\mathbf{p}=\left(2 m_{1} \pi, \ldots, 2 m_{n} \pi\right), m_{j} \in \mathbb{Z}$, and on the other hand there exists $\varepsilon_{0}>0$ such that $\|\mathbf{x}-\mathbf{z}\| \geq \varepsilon_{0}$ for any $\mathbf{z} \in L_{\mathbf{v}, \boldsymbol{\lambda}}$. Moreover, since $\mathbf{w} \in \overline{L_{\mathbf{v}, \boldsymbol{\lambda}}}$, we can locate $t_{1} \in \mathbb{R}$ and $\mathbf{q} \in \mathbb{Z}^{n}$ such that $\mathbf{w}=\mathbf{v}+t_{1} \boldsymbol{\lambda}+\mathbf{q}+\varepsilon$, for some $\varepsilon \in \mathbb{R}^{n}$ such that

$$
\|\varepsilon\|<\min \left\{\varepsilon_{0}, x_{i}, 2 \pi-x_{i}: i=1, \ldots, n\right\} .
$$

Thus $\mathbf{x}=\mathbf{v}+\left(t_{0}+t_{1}\right) \boldsymbol{\lambda}+\varepsilon+\mathbf{p}+\mathbf{q}$. Now, if we define $\mathbf{z}_{0}=\mathbf{v}+\left(t_{0}+t_{1}\right) \boldsymbol{\lambda}+\mathbf{p}+\mathbf{q}$, by taking (3.2) into account, we have that $\mathbf{z}_{0} \in L_{\mathbf{v}, \boldsymbol{\lambda}}$ and $\left\|\mathbf{x}-\mathbf{z}_{0}\right\|=\|\varepsilon\|<\varepsilon_{0}$, which represents a contradiction.

The lemma above (and its demonstration) is the key to prove the following result.

Proposition 13 Given two points $\mathbf{v} \in \mathbb{R}^{n}$ and $\mathbf{w} \in C$, and a vector $\boldsymbol{\lambda}=$ $\left(\lambda_{1}, \ldots, \lambda_{n}\right)$, then $\overline{L_{\mathbf{w}, \boldsymbol{\lambda}}}=\overline{L_{\mathbf{v}, \boldsymbol{\lambda}}}$ if and only if $\mathbf{w} \in \overline{L_{\mathbf{v}, \boldsymbol{\lambda}}}$.

Proof. Take $\overline{L_{\mathbf{w}, \boldsymbol{\lambda}}}=\overline{L_{\mathbf{v}, \boldsymbol{\lambda}}}$. Since $\mathbf{w} \in L_{\mathbf{w}, \boldsymbol{\lambda}}$, it is accomplished that $\mathbf{w} \in \overline{L_{\mathbf{w}, \boldsymbol{\lambda}}}=$ $\overline{L_{\mathbf{v}, \boldsymbol{\lambda}}}$. Conversely, let $\mathbf{w} \in \overline{L_{\mathbf{v}, \boldsymbol{\lambda}}}$. By Lemma 12, it is clear that $\overline{L_{\mathbf{w}, \boldsymbol{\lambda}}} \subset \overline{L_{\mathbf{v}, \boldsymbol{\lambda}}}$. We next prove by reductio ad absurdum that $\overline{L_{\mathbf{v}, \boldsymbol{\lambda}}} \subset \overline{L_{\mathbf{w}, \boldsymbol{\lambda}}}$. So, take $\mathbf{x} \in \overline{L_{\mathbf{v}, \boldsymbol{\lambda}}}$

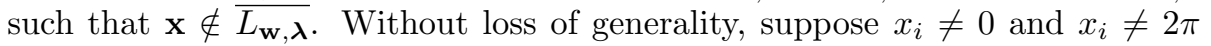
for each $i=1, \ldots, n$ ( $\overline{L_{\mathbf{v}, \boldsymbol{\lambda}}}$ is formed from affine subspaces). Also, take $d:=$ $\min \left\{\frac{x_{i}}{2}, \frac{2 \pi-x_{i}}{2}: i=1, \ldots, n\right\}$. Then there exists $\varepsilon_{0}>0$ such that $\|\mathbf{x}-\mathbf{z}\| \geq \varepsilon_{0}$ 
for any $\mathbf{z} \in L_{\mathbf{w}, \boldsymbol{\lambda}}$. Now, since $\mathbf{x} \in \overline{L_{\mathbf{v}, \boldsymbol{\lambda}}}$, we can write $\mathbf{x}=\mathbf{v}+t_{0} \boldsymbol{\lambda}+\mathbf{p}+\varepsilon$ for some $t_{0} \in \mathbb{R}, \mathbf{p}=\left(2 m_{1} \pi, \ldots, 2 m_{n} \pi\right), m_{j} \in \mathbb{Z}$, and $\varepsilon \in \mathbb{R}^{n}$ such that

$$
\|\varepsilon\|<\min \left\{\frac{\varepsilon_{0}}{2}, d\right\} .
$$

Moreover, since $\mathbf{w} \in \overline{L_{\mathbf{v}, \boldsymbol{\lambda}}}$, we can locate $t_{1} \in \mathbb{R}$ and $\mathbf{q} \in \mathbb{Z}^{n}$ such that $\mathbf{w}=\mathbf{v}+t_{1} \boldsymbol{\lambda}+\mathbf{q}+\tau$ for some $\tau \in \mathbb{R}^{n}$ satisfying

$$
\|\tau\|<\min \left\{\frac{\varepsilon_{0}}{2}, d\right\} .
$$

Then $\mathbf{x}=\mathbf{w}+\left(t_{0}-t_{1}\right) \boldsymbol{\lambda}+\varepsilon-\tau+\mathbf{p}-\mathbf{q}$. So, if we define $\mathbf{z}_{0}=\mathbf{w}+\left(t_{0}-t_{1}\right) \boldsymbol{\lambda}+\mathbf{p}-\mathbf{q}$, by taking (3.3) and (3.4) into account, we have that $\mathbf{z}_{0} \in L_{\mathbf{w}, \boldsymbol{\lambda}}$ and $\left\|\mathbf{x}-\mathbf{z}_{0}\right\|=$ $\|\varepsilon-\tau\|<\varepsilon_{0}$, which is a contradiction. Thus the result holds.

\section{The generator functions}

In this section we are going to handle a class of functions which will generate the images of every exponential polynomial $P(s)$ of type (1.1). The number of variables of these generator functions coincides with the number of coefficients of the associated exponential polynomials.

Definition 14 Given $n \in \mathbb{N}$, let $B=\left\{b_{1}, \ldots, b_{n}\right\}$ be a set of $n$ non-negative numbers. We define the generator function $g_{B}: \mathbb{R}^{n} \rightarrow \mathbb{C}$ associated with $B$ as

$$
g_{B}(\mathbf{x}):=\sum_{j=1}^{n} b_{j} e^{i x_{j}}, \mathbf{x}=\left(x_{1}, \ldots, x_{n}\right) \in \mathbb{R}^{n} .
$$

Remark 15 Every evaluation of an exponential polynomial of the form $P(s)=\sum_{j=1}^{n} a_{j} e^{\lambda_{j} s} \in \mathcal{P}_{\Lambda}$, where $\Lambda=\left\{\lambda_{1}, \lambda_{2}, \ldots, \lambda_{n}\right\}$ is a set of exponents, can be obtained from the generator functions by evaluating them on certain lines. Indeed, fixed $\sigma_{0} \in \mathbb{R}$, take $B_{P, \sigma_{0}}=\left\{\left|a_{1}\right| e^{\lambda_{1} \sigma_{0}},\left|a_{2}\right| e^{\lambda_{2} \sigma_{0}}, \ldots,\left|a_{n}\right| e^{\lambda_{n} \sigma_{0}}\right\}$ and note that

$$
g_{B_{P, \sigma_{0}}}\left(\arg a_{1}+t \lambda_{1}, \ldots, \arg a_{n}+t \lambda_{n}\right)=P\left(\sigma_{0}+i t\right) \forall t \in \mathbb{R} .
$$

It is clear that $g_{B}\left(x_{1}, \ldots, x_{n}\right)$ is $2 \pi$-periodic in each coordinate $x_{k}$, with $k=1, \ldots, n$. In this sense, this property prompts a structure which is similar to that of a crystal and connects these functions with the concepts introduced in previous sections.

For the following lemma, take the notation

$$
\operatorname{Img}\left(P\left(\sigma_{0}+i t\right)\right)=\left\{s \in \mathbb{C}: \exists t \in \mathbb{R} \text { such that } s=P\left(\sigma_{0}+i t\right)\right\}
$$

and

$$
\operatorname{Img}\left(g_{B}(A)\right)=\left\{s \in \mathbb{C}: \exists \mathbf{x} \in A \text { such that } s=g_{B}\left(\sigma_{0}, \mathbf{x}\right)\right\}
$$

for the set of values in the complex plane taken on by an exponential polynomial $P(\sigma+i t) \in \mathcal{P}_{\Lambda}$ and $g_{B}(A)$ for some $\sigma=\sigma_{0}$ and $A \subset \mathbb{R}^{n}$, respectively. Now remark 15 inspires the following lemma. 
Lemma 16 Given $n \in \mathbb{N}$ and $\Lambda=\left\{\lambda_{1}, \ldots, \lambda_{n}\right\}$ a set of $n$ exponents, let $P(s)=$ $\sum_{j=1}^{n} a_{j} e^{\lambda_{j} s} \in \mathcal{P}_{\Lambda}$. Then

$$
\operatorname{Img}\left(P\left(\sigma_{0}+i t\right)\right)=\operatorname{Img}\left(g_{B_{P, \sigma_{0}}}\left(L_{\mathbf{v}, \boldsymbol{\lambda}}\right)\right),
$$

where $\sigma_{0}$ is a real number, $\boldsymbol{\lambda}=\left(\lambda_{1}, \ldots, \lambda_{n}\right), \mathbf{v}=\left(\arg a_{1}, \ldots, \arg a_{n}\right)$ and $B_{P, \sigma_{0}}=\left\{\left|a_{1}\right| e^{\lambda_{1} \sigma_{0}}, \ldots,\left|a_{n}\right| e^{\lambda_{n} \sigma_{0}}\right\}$.

Proof. Given $\boldsymbol{\lambda}=\left(\lambda_{1}, \ldots, \lambda_{n}\right), P(s)=\sum_{j=1}^{n} a_{j} e^{\lambda_{j} s} \in \mathcal{P}_{\Lambda}$ and $\sigma_{0} \in \mathbb{R}$, let $B_{P, \sigma_{0}}=\left\{\left|a_{1}\right| e^{\lambda_{1} \sigma_{0}}, \ldots,\left|a_{n}\right| e^{\lambda_{n} \sigma_{0}}\right\}$. If $\mathbf{x}=\mathbf{v}+t^{*} \boldsymbol{\lambda}$, with $\mathbf{v}=\left(\arg a_{1}, \ldots, \arg a_{n}\right)$ and $t^{*} \in \mathbb{R}$, then by Remark 15 we have $g_{B_{P, \sigma_{0}}}\left(x_{1}, \ldots, x_{n}\right)=P\left(\sigma_{0}+i t^{*}\right)$. Hence, by varying $t^{*}$, it is satisfied

$$
\operatorname{Img}\left(P\left(\sigma_{0}+i t\right)\right)=\operatorname{Img}\left(g_{B_{P, \sigma_{0}}}\left(A_{\mathbf{v}, \boldsymbol{\lambda}}\right)\right),
$$

where $A_{\mathbf{v}, \boldsymbol{\lambda}}:=\left\{\mathbf{x} \in \mathbb{R}^{n}: \mathbf{x}=\mathbf{v}+t \boldsymbol{\lambda}, t \in \mathbb{R}\right\}$. Finally, since $L_{\mathbf{v}, \boldsymbol{\lambda}}=R\left(A_{\mathbf{v}, \boldsymbol{\lambda}}\right)$, with $R$ the reduction map taking each element $\left(y_{1}, \ldots, y_{n}\right) \in \mathbb{R}^{n}$ to its equivalent modulus in the period $[0,2 \pi)$, we deduce from periodicity of each coordinate $x_{k}$ that $\operatorname{Img}\left(g_{B_{P, \sigma_{0}}}\left(A_{\mathbf{v}, \boldsymbol{\lambda}}\right)\right)=\operatorname{Img}\left(g_{B_{P, \sigma_{0}}}\left(L_{\mathbf{v}, \boldsymbol{\lambda}}\right)\right)$, and the result follows.

As a consequence of the lemma above and the continuity of the function $g_{B}(\mathbf{x})$, the proof of the following result is clear.

Lemma 17 Given $n \in \mathbb{N}$ and $\Lambda=\left\{\lambda_{1}, \ldots, \lambda_{n}\right\}$ a set of $n$ exponents, let $P(s)=\sum_{j=1}^{n} a_{j} e^{\lambda_{j} s} \in \mathcal{P}_{\Lambda}$. Then

$$
\overline{\operatorname{Img}\left(P\left(\sigma_{0}+i t\right)\right)}=\overline{\operatorname{Img}\left(g_{B_{P, \sigma_{0}}}\left(\overline{L_{\mathbf{v}, \boldsymbol{\lambda}}}\right)\right)},
$$

where $\sigma_{0}$ is a real number, $\boldsymbol{\lambda}=\left(\lambda_{1}, \ldots, \lambda_{n}\right), \mathbf{v}=\left(\arg a_{1}, \ldots, \arg a_{n}\right)$ and $B_{P, \sigma_{0}}=\left\{\left|a_{1}\right| e^{\lambda_{1} \sigma_{0}}, \ldots,\left|a_{n}\right| e^{\lambda_{n} \sigma_{0}}\right\}$.

\section{Equivalent exponential polynomials}

We next introduce an equivalence relation on the classes $\mathcal{P}_{\Lambda}$, which will be our most valuable tool from now on.

Definition 18 Given $\Lambda=\left\{\lambda_{1}, \lambda_{2}, \ldots, \lambda_{n}\right\}$ a set of exponents, let $V$ be the $\mathbb{Q}$ vector space generated by $\Lambda$. Consider $P_{1}(s)=\sum_{j=1}^{n} a_{j} e^{\lambda_{j} s}$ and $P_{2}(s)=\sum_{j=1}^{n} b_{j} e^{\lambda_{j} s}$ two exponential polynomials in the class $\mathcal{P}_{\Lambda}$. We will say that $P_{1}$ is equivalent to $P_{2}$, and it will be denoted as $P_{1} \sim P_{2}$, if there exists $a \mathbb{Q}$-linear map $\psi: V \rightarrow \mathbb{R}$ such that

$$
b_{j}=a_{j} e^{i \psi\left(\lambda_{j}\right)}, \lambda_{j} \in \Lambda .
$$

It is immediate that $\sim$ is an equivalence relation (in fact, it coincides with that of $\left[9\right.$, Definition 3] for the case of the functions in the classes $\left.\mathcal{P}_{\Lambda}\right)$. The reader can observe that this equivalence relation is based on that of Bohr (see $[1$, p.173]) which was defined for general Dirichlet series and was characterized 
in terms of a completely multiplicative function [1, Theorem 8.12]. In fact, although Apostol does not use the concept of vectorial space in his book [1], it is plain that he considers the $\mathbb{Q}$-vector space generated by the sequence of exponents implicitly.

We next consider the following result which characterizes the notion of equivalent functions in $\mathcal{P}_{\Lambda}$ in terms of a basis of the $\mathbb{Q}$-vector space generated by $\left\{\lambda_{1}, \ldots, \lambda_{n}\right\}$ (compare with the definition given in $[1$, p.173] and with $[9$, Proposition 1]).

Lemma 19 Given $\Lambda$ a set of exponents, consider $P_{1}(s)=\sum_{j=1}^{n} a_{j} e^{\lambda_{j} s}$ and $P_{2}(s)=\sum_{j=1}^{n} b_{j} e^{\lambda_{j} s}$ two exponential polynomials in the class $\mathcal{P}_{\Lambda}$. Let $\mathbf{g}$ be the vector of the elements of a basis of the $\mathbb{Q}$-vector space generated by $\left\{\lambda_{1}, \ldots, \lambda_{n}\right\}$ and $\mathbf{r}_{j}$ the vector of rational components that verify $\lambda_{j}=<\mathbf{r}_{j}, \mathbf{g}>$ for each $j=1, \ldots, n$. Then $P_{1}$ is equivalent to $P_{2}$ if and only if there exists some $\mathbf{x}_{0} \in \mathbb{R}^{k}$ such that $b_{j}=a_{j} e^{<\mathbf{r}_{j}, \mathbf{x}_{0}>i}$ for every $j=1, \ldots, n$.

Proof. Let $V$ be the $\mathbb{Q}$-vector space generated by $\Lambda$ and $\mathbf{g}=\left(g_{1}, \ldots, g_{k}\right)$ the vector of the elements of a basis of $V$. If $P_{1} \sim P_{2}$, by Definition 18 there exists a $\mathbb{Q}$-linear map $\psi: V \rightarrow \mathbb{R}$ such that $b_{j}=a_{j} e^{i \psi\left(\lambda_{j}\right)}, j=1,2 \ldots, n$. Hence $b_{j}=a_{j} e^{i \sum_{l=1}^{k} r_{j, l} \psi\left(g_{l}\right)}, j=1, \ldots, n$ or, equivalently,

$$
b_{j}=a_{j} e^{i<\mathbf{r}_{j}, \mathbf{x}_{0}>}, j=1, \ldots, n
$$

with $\mathbf{x}_{0}:=\left(\psi\left(g_{1}\right), \ldots, \psi\left(g_{k}\right)\right)$. Conversely, suppose the existence of a vector $\mathbf{x}_{0}=\left(x_{0,1}, \ldots, x_{0, k}\right) \in \mathbb{R}^{k}$ such that $b_{j}=a_{j} e^{<\mathbf{r}_{j}, \mathbf{x}_{0}>i}, j=1, \ldots, n$. Then a $\mathbb{Q}$-linear map $\psi: V \rightarrow \mathbb{R}$ can be defined from $\psi\left(g_{l}\right):=x_{0, l}, l=1, \ldots, k$. Hence

$$
\psi\left(\lambda_{j}\right)=\sum_{l=1}^{k} r_{j, l} \psi\left(g_{l}\right)=<\mathbf{r}_{j}, \mathbf{x}_{0}>, j=1, \ldots, n
$$

and the result follows.

The next propositions provide us the connection between equivalent functions in terms of the sets $L_{\mathbf{v}, \boldsymbol{\lambda}}$.

Proposition 20 Given $\Lambda=\left\{\lambda_{1}, \lambda_{2}, \ldots, \lambda_{n}\right\}$ a set of $n \geq 2$ exponents, let $P_{1}(s)=\sum_{j=1}^{n} a_{j} e^{\lambda_{j} s}$ and $P_{2}(s)=\sum_{j=1}^{n} b_{j} e^{\lambda_{j} s}$ be two exponential polynomials in $\mathcal{P}_{\Lambda}$ such that $P_{1} \sim P_{2}$. Consider $\mathbf{v}=\left(\arg a_{1}, \arg a_{2}, \ldots, \arg a_{n}\right)$, $\mathbf{w}=\left(\arg b_{1}, \arg b_{2}, \ldots, \arg b_{n}\right)$ and $\boldsymbol{\lambda}=\left(\lambda_{1}, \lambda_{2}, \ldots, \lambda_{n}\right)$. Then $\overline{L_{\mathbf{v}, \boldsymbol{\lambda}}}=\overline{L_{\mathbf{w}, \boldsymbol{\lambda}}}$.

Proof. Let $k$ be the cardinal of any basis of the $\mathbb{Q}$-vector space generated by $\left\{\lambda_{1}, \ldots, \lambda_{n}\right\}$. Suppose that $\{1, \ldots, n\}=I_{1} \cup I_{2}$, with $I_{1} \cap I_{2}=\emptyset$ and $\sharp I_{1}=k$, where the set $\left\{\lambda_{j}: j \in I_{1}\right\}$ is linearly independent over the rational numbers and $\lambda_{j}=\sum_{l \in I_{1}} r_{j, l} \lambda_{l}, j \in I_{2}$, for some $r_{j, l} \in \mathbb{Q}, j \in I_{2}, l \in I_{1}$. Note first that, from the equivalence of $P_{1}(s)$ and $P_{2}(s)$, we deduce the existence of some $\mathbf{z} \in \mathbb{R}^{k}$ such that $b_{j}=a_{j} e^{<\mathbf{r}_{j}, \mathbf{z}>i}, j=1,2, \ldots, n$ and hence there exist $m_{j} \in \mathbb{Z}$ such that

$$
\arg b_{j}=\arg a_{j}+<\mathbf{r}_{j}, \mathbf{z}>+2 \pi m_{j}, j=1,2, \ldots, n,
$$


or equivalently

$$
w_{j}=v_{j}+y_{j}+2 \pi m_{j}, j=1,2, \ldots, n,
$$

where $y_{j}:=<\mathbf{r}_{j}, \mathbf{z}>$. By Proposition 9 , there exist $m_{1}, m_{2} \in \mathbb{N}$ such that

$$
\overline{L_{\mathbf{v}, \boldsymbol{\lambda}}}=\left(\bigcup_{j=1}^{m_{1}} A_{j}\right) \cap\left([0,2 \pi] \times \cdots^{n)} \times[0,2 \pi]\right)
$$

and

$$
\overline{L_{\mathbf{v}+\mathbf{y}, \boldsymbol{\lambda}}}=\left(\bigcup_{j=1}^{m_{2}} B_{j}\right) \cap\left([0,2 \pi] \times \cdots^{n)} \times[0,2 \pi]\right),
$$

for some $d$-dimensional affine subspaces $A_{j}, B_{j}$ in $\mathbb{R}^{n}$, where $d \leq k$. Moreover, the subspaces $A_{j}, j=1, \ldots, m_{1}$ are determined by equations (3.1), which are

$$
x_{j}=\left(v_{j}-\sum_{l \in I_{1}} r_{j, l} v_{l}\right)+\sum_{l \in I_{1}} r_{j, l} x_{l}, j \in I_{2},
$$

and analogously the subspaces $B_{j}, j=1, \ldots, m_{2}$ are determined by equations

$$
x_{j}=\left(v_{j}+y_{j}-\sum_{l \in I_{1}} r_{j, l}\left(v_{l}+y_{l}\right)\right)+\sum_{l \in I_{1}} r_{j, l} x_{l}, j \in I_{2} .
$$

However, we next show that (5.1) and (5.2) are equal. Indeed, note first that, if $j \in I_{2}$, we have $r_{j, l}=0$ for each $l \in I_{1}$ (the numbers $\left\{\lambda_{j}: j \in I_{2}\right\}$ are rationally dependent). Secondly, if $l \in I_{1}$, then $r_{l, i}=1$ for $i=l$ and $r_{l, i}=0$ for $i \neq l$. Hence

$$
\begin{gathered}
y_{j}=<\mathbf{r}_{j}, \mathbf{z}>=\sum_{l=1}^{n} r_{j, l} z_{l}=\sum_{l \in I_{1}} r_{j, l} z_{l}, j \in I_{2}, \\
y_{l}=<\mathbf{r}_{l}, \mathbf{z}>=\sum_{i=1}^{n} r_{l, i} z_{i}=z_{l}, l \in I_{1},
\end{gathered}
$$

and then

$$
\sum_{l \in I_{1}} r_{j, l} y_{l}=\sum_{l \in I_{1}} r_{j, l} z_{l}, j \in I_{2} .
$$

Consequently,

$$
v_{j}-\sum_{l \in I_{1}} r_{j, l} v_{l}=v_{j}+y_{j}-\sum_{l \in I_{1}} r_{j, l}\left(v_{l}+y_{l}\right), j \in I_{2}
$$

and thus (5.1) and (5.2) are equal, which yields that $\overline{L_{\mathbf{v}, \boldsymbol{\lambda}}}=\overline{L_{\mathbf{v}+\mathbf{y}, \boldsymbol{\lambda}}}$. Finally, since $L_{\mathbf{v}+\mathbf{y}, \boldsymbol{\lambda}}=L_{\mathbf{w}, \boldsymbol{\lambda}}$, we conclude that $\overline{L_{\mathbf{v}, \boldsymbol{\lambda}}}=\overline{L_{\mathbf{w}, \boldsymbol{\lambda}}}$.

It is worth noting the connection between Bohr's equivalence theorem [1, p.178] and part ii) of our next result (compare also with the results of the recent paper [10]). 
Proposition 21 Given $\Lambda=\left\{\lambda_{1}, \lambda_{2}, \ldots, \lambda_{n}\right\}$ a set of $n \geq 2$ exponents, let $P_{1}(s)=\sum_{j=1}^{n} a_{j} e^{\lambda_{j} s}$ and $P_{2}(s)=\sum_{j=1}^{n} b_{j} e^{\lambda_{j} s}$ be two equivalent exponential polynomials in $\mathcal{P}_{\Lambda}$. Consider the vectors $\mathbf{v}=\left(\arg a_{1}, \arg a_{2}, \ldots, \arg a_{n}\right), \mathbf{w}=$ $\left(\arg b_{1}, \arg b_{2}, \ldots, \arg b_{n}\right)$, and the point $\boldsymbol{\lambda}=\left(\lambda_{1}, \lambda_{2}, \ldots, \lambda_{n}\right)$. Given $\sigma_{0} \in \mathbb{R}$, take the sets $B_{P_{1}, \sigma_{0}}=\left\{\left|a_{1}\right| e^{\lambda_{1} \sigma_{0}},\left|a_{2}\right| e^{\lambda_{2} \sigma_{0}}, \ldots,\left|a_{n}\right| e^{\lambda_{n} \sigma_{0}}\right\}$ and $B_{P_{2}, \sigma_{0}}=\left\{\left|b_{1}\right| e^{\lambda_{1} \sigma_{0}},\left|b_{2}\right| e^{\lambda_{2} \sigma_{0}}, \ldots,\left|b_{n}\right| e^{\lambda_{n} \sigma_{0}}\right\}$. Then it is accomplished that

i) $\operatorname{Img}\left(P_{2}\left(\sigma_{0}+i t\right)\right)=\operatorname{Img}\left(g_{B_{P_{1}, \sigma_{0}}}\left(L_{\mathbf{w}, \boldsymbol{\lambda}}\right)\right)$.

ii) $\overline{\operatorname{Img}\left(P_{1}\left(\sigma_{0}+i t\right)\right)}=\overline{\operatorname{Img}\left(P_{2}\left(\sigma_{0}+i t\right)\right)}$.

iii) $\overline{\operatorname{Img}\left(g_{B_{P_{1}, \sigma_{0}}}\left(L_{\mathbf{v}, \boldsymbol{\lambda}}\right)\right)}=\overline{\operatorname{Img}\left(g_{B_{P_{1}, \sigma_{0}}}\left(L_{\mathbf{w}, \boldsymbol{\lambda}}\right)\right)}=\overline{\operatorname{Img}\left(g_{B_{P_{2}, \sigma_{0}}}\left(L_{\mathbf{w}, \boldsymbol{\lambda}}\right)\right)}$.

Proof. Note first that, from the equivalence of $P_{1}(s)$ and $P_{2}(s)$, we can assure the existence of some $\mathbf{z} \in \mathbb{R}^{k}$ satisfying $b_{j}=a_{j} e^{<\mathbf{r}_{j}, \mathbf{z}>i}, j=1,2, \ldots, n$ and hence there exist $m_{j} \in \mathbb{Z}$ such that

$$
\arg b_{j}=\arg a_{j}+<\mathbf{r}_{j}, \mathbf{z}>+2 \pi m_{j}, j=1,2, \ldots, n,
$$

or equivalently

$$
w_{j}=v_{j}+y_{j}+2 \pi m_{j}, j=1,2, \ldots, n,
$$

where $y_{j}:=<\mathbf{r}_{j}, \mathbf{z}>$.

i) Given $\sigma_{0} \in \mathbb{R}$, take $B_{P_{1}, \sigma_{0}}=\left\{\left|a_{1}\right| e^{\lambda_{1} \sigma_{0}},\left|a_{2}\right| e^{\lambda_{2} \sigma_{0}}, \ldots,\left|a_{n}\right| e^{\lambda_{n} \sigma_{0}}\right\}$. Observe that

$$
\operatorname{Img}\left(P_{1}\left(\sigma_{0}+i t\right)\right)=\operatorname{Img}\left(g_{B_{P_{1}, \sigma_{0}}}\left(L_{\mathbf{v}, \boldsymbol{\lambda}}\right)\right),
$$

follows from Lemma 16. On the other hand, we have

$$
\begin{gathered}
g_{B_{P_{1}, \sigma_{0}}}\left(t \lambda_{1}+v_{1}+y_{1}, t \lambda_{2}+v_{2}+y_{2}, \ldots, t \lambda_{k}+v_{k}+y_{k}, \ldots\right)= \\
=\sum_{j=1}^{n}\left|a_{j}\right| e^{\lambda_{j} \sigma_{0}} e^{i\left(t \lambda_{j}+v_{j}+y_{j}\right)}=\sum_{j=1}^{n} a_{j} e^{\lambda_{j}\left(\sigma_{0}+i t\right)} e^{i y_{j}}= \\
=\sum_{j=1}^{n} b_{j} e^{\lambda_{j}\left(\sigma_{0}+i t\right)}=P_{2}\left(\sigma_{0}+i t\right) \forall t \in \mathbb{R} .
\end{gathered}
$$

Hence, as in Lemma 16, we have $\operatorname{Img}\left(P_{2}\left(\sigma_{0}+i t\right)\right)=\operatorname{Img}\left(g_{B_{P_{1}, \sigma_{0}}}\left(L_{\mathbf{v}+\mathbf{y}, \boldsymbol{\lambda}}\right)\right)$. Now, by (5.3) and remark 3 , note that $L_{\mathbf{v}+\mathbf{y}, \boldsymbol{\lambda}}=L_{\mathbf{w}, \boldsymbol{\lambda}}$ and hence $\operatorname{Img}\left(P_{2}\left(\sigma_{0}+i t\right)\right)=\operatorname{Img}\left(g_{B_{P_{1}, \sigma_{0}}}\left(L_{\mathbf{w}, \boldsymbol{\lambda}}\right)\right)$.

ii) We deduce from Lemma $17,(5.4)$ and point i) that

$$
\begin{aligned}
& \overline{\operatorname{Img}\left(P_{1}\left(\sigma_{0}+i t\right)\right)}=\overline{\operatorname{Img}\left(g_{B_{P_{1}, \sigma_{0}}}\left(\overline{L_{\mathbf{v}, \boldsymbol{\lambda}}}\right)\right)}= \\
& =\overline{\operatorname{Img}\left(g_{B_{P_{1}, \sigma_{0}}}\left(\overline{\left.L_{\mathbf{w}, \boldsymbol{\mu}}\right)}\right)\right.}=\overline{\operatorname{Img}\left(P_{2}\left(\sigma_{0}+i t\right)\right)} .
\end{aligned}
$$

iii) It follows from i) and ii).

As might be suspected from Proposition 6, two arbitrary points in $\mathbb{R}^{n}$ provide two equivalent exponential polynomials when the set $\Lambda$ is linearly independent over the rationals. Indeed, it is also a consequence of the following result. 
Proposition 22 Let $\Lambda=\left\{\lambda_{1}, \lambda_{2}, \ldots, \lambda_{n}\right\}$ be a set of $n \geq 2$ real numbers which are linearly independent over the rationals and $P_{1}(s)=\sum_{j=1}^{n} a_{j} e^{\lambda_{j} s}$, $P_{2}(s)=\sum_{j=1}^{n} b_{j} e^{\lambda_{j} s}$ be two exponential polynomials in $\mathcal{P}_{\Lambda}$. Then $P_{1} \sim P_{2}$ if and only if $\left|a_{j}\right|=\left|b_{j}\right|$ for each $j=1,2, \ldots, n$.

Proof. If $P_{1} \sim P_{2}$, then Lemma 19 assures the existence of $\mathbf{x}_{0} \in \mathbb{R}^{n}$ such that $b_{j}=a_{j} e^{i\left\langle\mathbf{r}_{j}, \mathbf{x}_{0}\right\rangle}$ for each $j=1, \ldots, n$, which proves $\left|a_{j}\right|=\left|b_{j}\right|$ for each $j=1,2, \ldots, n$. Conversely, if $\left|a_{j}\right|=\left|b_{j}\right|$ for each $j=1,2, \ldots, n$, then there exists a vector $\theta=\left(\theta_{1}, \theta_{2}, \ldots, \theta_{n}\right) \in[0,2 \pi]^{n}$ such that $b_{j}=a_{j} e^{i \theta_{j}}$ for each $j=1,2, \ldots, n$. Since $\Lambda$ is linearly independent over the rationals, take $\mathbf{g}=\left(\lambda_{1}, \lambda_{2}, \ldots, \lambda_{n}\right)$ as the vector of the elements of a basis of the $\mathbb{Q}$-vector space generated by $\Lambda$, then the vectors $\mathbf{r}_{j}$ of rational components so that $\left\langle\mathbf{r}_{j}, \mathbf{g}\right\rangle=\lambda_{j}$ verify $\mathbf{r}_{j, l}=0$ if $j \neq l$ and $\mathbf{r}_{j, l}=1$ if $j=l$. Hence $b_{j}=a_{j} e^{i \theta_{j}}=a_{j} e^{i<\mathbf{r}_{j}, \theta>}$ for each $j=1,2, \ldots, n$, which means that $P_{1} \sim P_{2}$.

Remark 23 As a consequence of the result above, if $\boldsymbol{\lambda}=\left(\lambda_{1}, \lambda_{2}, \ldots, \lambda_{n}\right)$, $\mathbf{v}=\left(v_{1}, v_{2}, \ldots, v_{n}\right)$ and $\mathbf{w}=\left(w_{1}, w_{2}, \ldots, w_{n}\right)$ are in $\mathbb{R}^{n}$, with $\Lambda=\left\{\lambda_{1}, \lambda_{2}, \ldots, \lambda_{n}\right\}$ a set of $n$ real numbers which are linearly independent over the rationals, then $L_{\mathbf{v}, \boldsymbol{\lambda}}$ and $L_{\mathbf{w}, \boldsymbol{\lambda}}$ provide two exponential polynomials which are equivalent in $\mathcal{P}_{\Lambda}$. Indeed, without loss of generality, suppose that $v_{j}, w_{j} \in[0,2 \pi)$ and take

$$
P_{1}(s)=\sum_{j=1}^{n} a_{j} e^{\lambda_{j} s} \in \mathcal{P}_{\Lambda}, P_{2}(s)=\sum_{j=1}^{n} b_{j} e^{\lambda_{j} s} \in \mathcal{P}_{\Lambda},
$$

with $a_{j}, b_{j} \in \mathbb{C}$ such that $\left|a_{j}\right|=\left|b_{j}\right|$, arg $a_{j}=v_{j}$ and $\arg b_{j}=w_{j}$ for each $j$. Then $P_{1} \sim P_{2}$ and, fixed $\sigma_{0} \in \mathbb{R}$, we have

$$
\operatorname{Img}\left(P_{1}\left(\sigma_{0}+i t\right)\right)=\operatorname{Img}\left(g_{B_{P_{1}, \sigma_{0}}}\left(L_{\mathbf{v}, \boldsymbol{\lambda}}\right)\right)
$$

and

$$
\operatorname{Img}\left(P_{2}\left(\sigma_{0}+i t\right)\right)=\operatorname{Img}\left(g_{B_{P_{1}, \sigma_{0}}}\left(L_{\mathbf{w}, \boldsymbol{\lambda}}\right)\right),
$$

where $B_{P_{1}, \sigma_{0}}=\left\{\left|a_{1}\right| e^{\lambda_{1} \sigma_{0}},\left|a_{2}\right| e^{\lambda_{2} \sigma_{0}}, \ldots,\left|a_{n}\right| e^{\lambda_{n} \sigma_{0}}\right\}$.

It is plain that, in general, the remark above is not true when the elements of $\Lambda$ are not linearly independent over the rationals. In that case, in geometrical terms, we pointed in Remark 10 that the sets $L_{\mathbf{v}, \boldsymbol{\lambda}}$ and $L_{\mathbf{w}, \boldsymbol{\lambda}}$ provide parallel affine subspaces for any $\mathbf{v}$ and $\mathbf{w}$ in $\mathbb{R}^{n}$ with $n$ the number of elements of $\Lambda$.

In this respect, without the restriction of $\mathbb{Q}$-linear independence of the frequencies, we next prove that the converse of Proposition 20 is also true.

Theorem 24 Given $\Lambda=\left\{\lambda_{1}, \lambda_{2}, \ldots, \lambda_{n}\right\}$ is a set of $n \geq 2$ exponents, let $P_{1}(s)=\sum_{j=1}^{n} a_{j} e^{\lambda_{j} s}$ and $P_{2}(s)=\sum_{j=1}^{n} b_{j} e^{\lambda_{j} s}$ be two exponential polynomials in $\mathcal{P}_{\Lambda}$ with $\left|a_{j}\right|=\left|b_{j}\right|$ for each $j=1,2, \ldots, n$. Consider the vectors $\mathbf{v}=\left(\arg a_{1}, \arg a_{2}, \ldots, \arg a_{n}\right), \mathbf{w}=\left(\arg b_{1}, \arg b_{2}, \ldots, \arg b_{n}\right)$, and the point $\boldsymbol{\lambda}=\left(\lambda_{1}, \lambda_{2}, \ldots, \lambda_{n}\right)$. Then $P_{1} \sim P_{2}$ if and only if $\overline{L_{\mathbf{v}, \boldsymbol{\lambda}}}=\overline{L_{\mathbf{w}, \boldsymbol{\lambda}}}$. 
Proof. Let $k$ be the cardinal of any basis of the $\mathbb{Q}$-vector space generated by $\Lambda$. Suppose that $\{1, \ldots, n\}=I_{1} \cup I_{2}$, with $I_{1} \cap I_{2}=\emptyset$ and $\sharp I_{1}=k$, where the set $\left\{\lambda_{j}: j \in I_{1}\right\}$ is linearly independent over the rational numbers and $\lambda_{j}=\sum_{l \in I_{1}} r_{j, l} \lambda_{l}, j \in I_{2}$ for some $r_{j, l} \in \mathbb{Q}, j \in I_{2}$ and $l \in I_{1}$. The direct implication is proved in Proposition 20. Now, suppose that $\overline{L_{\mathbf{v}, \boldsymbol{\lambda}}}=\overline{L_{\mathbf{w}, \boldsymbol{\lambda}}}$, then we deduce from Proposition 9 that there exists $m \in \mathbb{N}$ such that

$$
\overline{L_{\mathbf{v}, \boldsymbol{\lambda}}}=\overline{L_{\mathbf{w}, \boldsymbol{\lambda}}}=\left(\bigcup_{j=1}^{m} A_{j}\right) \cap\left([0,2 \pi] \times \cdots^{n)} \times[0,2 \pi]\right),
$$

for some $d$-dimensional affine subspaces $A_{j}$ in $\mathbb{R}^{n}, j=1,2, \ldots, m$, where $d \leq k$. Moreover, the subspaces $A_{j}, j=1, \ldots, m$ are determined by equations (3.1), which are

$$
x_{j}=\left(v_{j}-\sum_{l \in I_{1}} r_{j, l} v_{l}\right)+\sum_{l \in I_{1}} r_{j, l} x_{l}, j \in I_{2},
$$

and they generate the same subspaces as those of the equations

$$
x_{j}=\left(w_{j}-\sum_{l \in I_{1}} r_{j, l} w_{l}\right)+\sum_{l \in I_{1}} r_{j, l} x_{l}, j \in I_{2},
$$

Hence $w_{j}-v_{j}=\sum_{l \in I_{1}} r_{j, l}\left(w_{l}-v_{l}\right)+2 \pi p_{j}$, for some $p_{j} \in \mathbb{Z}, j \in I_{2}$. Recall that if $j \in I_{2}$, we have $r_{j, i}=0$ for each $i \in I_{2}$ (the numbers $\left\{\lambda_{j}: j \in I_{2}\right\}$ are rationally dependent). Moreover, if $j \in I_{1}$, then $r_{j, i}=1$ for $i=j$ and $r_{j, i}=0$ for $i \neq j$. Thus we have

$$
w_{j}-v_{j}=<\mathbf{r}_{j}, \mathbf{w}-\mathbf{v}>+2 \pi p_{j}, j=1, \ldots, n,
$$

where $p_{j}:=0$ for each $j \in I_{1}$. Now, we deduce from (5.5) that

$$
b_{j}=\left|b_{j}\right| e^{i w_{j}}=\left|b_{j}\right| e^{i\left(v_{j}+<\mathbf{r}_{j}, \mathbf{w}-\mathbf{v}>+2 \pi p_{j}\right)}=a_{j} e^{<\mathbf{r}_{j}, \mathbf{w}-\mathbf{v}>i}, j=1, \ldots, n,
$$

which means that $P_{2}$ is equivalent to $P_{1}$.

Example 25 Consider

$$
f_{1}(s)=e^{s \log 2}+e^{s \log 3}+e^{s\left(\frac{\log 2}{2}+\frac{\log 3}{2}\right)}
$$

and

$$
f_{2}(s)=-e^{s \log 2}-e^{s \log 3}+e^{s\left(\frac{\log 2}{2}+\frac{\log 3}{2}\right)} .
$$

It is not difficult to prove that $f_{1} \sim f_{2}$. Moreover, by Theorem 24, it is equivalent to the equality $\overline{L_{\mathbf{v}, \boldsymbol{\lambda}}}=\overline{L_{\mathbf{w}, \boldsymbol{\lambda}}}$, where $\mathbf{v}=(0,0,0), \mathbf{w}=(\pi, \pi, 0)$ and $\boldsymbol{\lambda}=\left(\log 2, \log 3, \frac{\log 2}{2}+\frac{\log 3}{2}\right)$. Figure 4 presents an approximation to the sets $L_{\mathbf{v}, \boldsymbol{\lambda}}$ and $L_{\mathbf{w}, \boldsymbol{\lambda}}$.

Corollary 26 Given $\boldsymbol{\lambda}=\left(\lambda_{1}, \lambda_{2}, \ldots, \lambda_{n}\right)$ the vector of a set $\Lambda$ of $n \geq 2$ exponents, let $P_{1}(s)=\sum_{j=1}^{n} a_{j} e^{\lambda_{j} s}$ and $P_{2}(s)=\sum_{j=1}^{n} b_{j} e^{\lambda_{j} s}$ be two exponential polynomials in $\mathcal{P}_{\Lambda}$ such that $\left|a_{j}\right|=\left|b_{j}\right|$ for every $j=1, \ldots, n$. Consider $\mathbf{v}=\left(\arg a_{1}, \arg a_{2}, \ldots, \arg a_{n}\right)$ and $\mathbf{w}=\left(\arg b_{1}, \arg b_{2}, \ldots, \arg b_{n}\right)$. Then $P_{1}(s)$ and $P_{2}(s)$ are equivalent if and only if $\mathbf{w} \in \overline{L_{\mathbf{v}, \boldsymbol{\lambda}}}$.

Proof. The result follows easily from Proposition 13 and Theorem 24. 


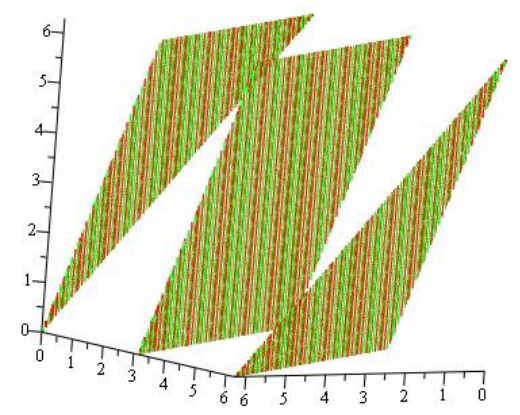

Figure 4: Approximation to the sets $L_{\mathbf{v}, \boldsymbol{\lambda}}$ (red) and $L_{\mathbf{w}, \boldsymbol{\lambda}}$ (green) of Example 25.

\section{Applications to the convergence of exponen- tial polynomials}

From now on, we will consider that the classes of exponential polynomials $\mathcal{P}_{\Lambda}$ are endowed with the topology of uniform convergence on every reduced vertical strip of $\mathbb{C}$.

As it was said in the introduction, Bochner's property consisting of the relative compactness of the set $\{f(t+\tau)\}, \tau \in \mathbb{R}$, associated with an almost periodic function $f$, is a characteristic feature of almost periodicity in the sense of Bohr. In this respect, this section aims to prove from our geometrical approach that every sequence of translates of an exponential polynomial has a subsequence that converges uniformly to an exponential polynomial which is equivalent to it. With this purpose, based on this approach, we next give an alternative proof of [9, Proposition 2].

Proposition 27 Let $\Lambda$ be a set of $n \geq 2$ exponents and $\mathcal{G}$ an equivalence class in $\mathcal{P}_{\Lambda} / \sim$. Then $\mathcal{G}$ is sequentially compact.

Proof. Given $\Lambda=\left\{\lambda_{1}, \lambda_{2}, \ldots, \lambda_{n}\right\}$, let $\left\{P_{l}\right\}_{l \geq 1}$ be a sequence in an equivalence class $\mathcal{G}$ in $\mathcal{P}_{\Lambda} / \sim$. Take $\boldsymbol{\lambda}=\left(\lambda_{1}, \lambda_{2}, \ldots, \lambda_{n}\right), P_{l}(s)=\sum_{j=1}^{n} a_{l, j} e^{\lambda_{j} s}$ and $\mathbf{v}_{l}=\left(\arg a_{l, 1}, \ldots, \arg a_{l, n}\right), l=1,2 \ldots$ By Proposition 21, we have that $\overline{L_{\mathbf{v}_{l}, \boldsymbol{\lambda}}}=\overline{L_{\mathbf{v}_{m}, \boldsymbol{\lambda}}}$ for any $l, m \geq 1$. Furthermore $\left|a_{l, j}\right|=\left|a_{m, j}\right|$ for any $l, m \in \mathbb{N}$ and $j=1, \ldots, n$. Also, note that $\left\{\mathbf{v}_{l}\right\}_{l \geq 1}$ is a sequence of points included in the origin cell $C$ (each one of them in $L_{\mathbf{v}_{l}, \boldsymbol{\lambda}}$ ) and hence it has a limit point, say

$\mathbf{w} \in \overline{L_{\mathbf{v}_{1}, \boldsymbol{\lambda}}}$. By abuse of language, let $\left\{\mathbf{v}_{l}\right\}_{l \geq 1}$ denote a subsequence converging to $\mathbf{w}$; that is, given $\varepsilon>0$, there exists $l_{0}$ such that $\left\|\mathbf{v}_{l}-\mathbf{w}\right\|<\varepsilon$ for any $l \geq l_{0}$, which yields

$$
\left|\mathbf{v}_{l, j}-\mathbf{w}_{j}\right|<\varepsilon \text { for each } l \geq l_{0} \text { and } j=1, \ldots, n \text {. }
$$


Now, by taking into account Proposition 13, we have $\overline{L_{\mathbf{v}_{1}, \boldsymbol{\lambda}}}=\overline{L_{\mathbf{w}, \boldsymbol{\lambda}}}$. Consequently, by Theorem 24, we conclude that $Q(s):=\sum_{j=1}^{n}\left|a_{1, j}\right| e^{i w_{j}} e^{\lambda_{j} s}$ is equivalent to $P_{1}$ and hence is included in $\mathcal{G}$. We next prove that there exists a subsequence in $\left\{P_{l}\right\}_{l \geq 1}$ convergent to $Q(s)$ with the topology of uniform convergence on $\mathbb{C}$. Indeed, By (6.1), there exist real numbers $\theta_{l, j}$, with $\left|\theta_{l, j}\right|<\varepsilon$, such that for each $l \geq l_{0}$ it is accomplished

$$
\left|e^{i w_{j}}-e^{i \arg a_{l, j}}\right|=\left|e^{i\left(\arg a_{l, j}+\theta_{l, j}\right)}-e^{i \arg a_{l, j}}\right|=\left|e^{i \theta_{l, j}}-1\right| \leq\left|\theta_{l, j}\right|<\varepsilon
$$

Now, fixed a reduced vertical strip $U \subset \mathbb{C}$ and $s=\sigma+i t$, for each $l \geq l_{0}$ we have

$$
\begin{gathered}
\left|Q(s)-P_{l}(s)\right|=\left|\sum_{j=1}^{n}\right| a_{1, j}\left|e^{i w_{j}} e^{\lambda_{j} s}-\sum_{j=1}^{n}\right| a_{l, j}\left|e^{i \arg a_{l, j}} e^{\lambda_{j} s}\right|= \\
=\left|\sum_{j=1}^{n}\right| a_{1, j}\left|e^{\lambda_{j} s}\left(e^{i w_{j}}-e^{i \arg a_{l, j}}\right)\right| \leq \\
\leq \sum_{j=1}^{n}\left|a_{1, j}\right| e^{\lambda_{j} \sigma}\left|e^{i w_{j}}-e^{i \arg a_{l, j}}\right|<\varepsilon \sum_{j=1}^{n}\left|a_{1, j}\right| e^{\lambda_{j} \sigma} .
\end{gathered}
$$

Thus the result follows.

As exponential polynomials in $\mathcal{P}_{\Lambda}$ are regarded as members of a metric space, we recall that sequential compactness is the same as compactness (in the topology induced by the metric), and it implies being closed.

Now, since the vertical translates of an exponential polynomial $P(s)$ are in the same equivalence class as $P(s)$, the following important corollary is immediate (compare with $[9$, Theorem 4$]$ ).

Corollary 28 Given $\Lambda$ a set of exponents and $P \in \mathcal{P}_{\Lambda}$, then the family of its vertical translates $\{P(s+i t)\}, t \in \mathbb{R}$, has a subsequence that converges uniformly to an equivalent function.

Finally, another important consequence of Proposition 27 is given in the following result (compare with [9, Corollary 5]).

Corollary 29 Let $\Lambda$ be a set of $n \geq 2$ exponents and $\mathcal{G}$ an equivalence class in $\mathcal{P}_{\Lambda} / \sim$. Given two arbitrary exponential polynomials $P(s)$ and $Q(s)$ in $\mathcal{G}$, there exists a sequence of vertical translations of $P(s)$ converging unifomly to $Q(s)$.

Proof. Given $\boldsymbol{\lambda}=\left(\lambda_{1}, \lambda_{2}, \ldots, \lambda_{n}\right)$, take $P(s)=\sum_{j=1}^{n} a_{j} e^{\lambda_{j} s}, Q(s)=\sum_{j=1}^{n} b_{j} e^{\lambda_{j} s}$, $\mathbf{v}=\left(\arg a_{1}, \ldots, \arg a_{n}\right)$ and $\mathbf{w}=\left(\arg b_{1}, \ldots, \arg b_{n}\right)$. By Proposition 21, we have that $\overline{L_{\mathbf{v}, \boldsymbol{\lambda}}}=\overline{L_{\mathbf{w}, \boldsymbol{\lambda}}}$. Hence $\mathbf{w} \in \overline{L_{\mathbf{v}, \boldsymbol{\lambda}}}$ and thus there exists a sequence $\left\{\mathbf{v}_{l}\right\}_{l \geq 1} \subset L_{\mathbf{v}, \boldsymbol{\lambda}}$ converging to $\mathbf{w}$. Now we just need to introduce this sequence in the proof of Proposition 27 to get the result 
More generally, consider the classes of exponential polynomials $\mathcal{P}_{\Lambda}$ endowed with a certain topology on every reduced strip $U$ of $\mathbb{C}$ induced by a metric $\|\cdot\|_{G}$ satisfying $\left\|\sum_{j=1}^{n} a_{j} e^{\lambda_{j} s}\right\|_{G} \leq \sum_{j=1}^{n}\left|a_{j}\right| e^{\lambda_{j} \sigma}$ for any $\sigma \in U$. Then it is easy to see that the proof of Proposition 27 works for this topology and, hence, the results of this section can be extended to this case. The topology of uniform convergence on every reduced vertical strip of $\mathbb{C}$ is an specific example, but many other different examples can be found (for instance, by virtue of $[3, \mathrm{p}$. 73]).

Acknowledgements. The first author's research was partially supported by PGC2018-097960-B-C22 (MCIU/AEI/ERDF, UE)

\section{References}

[1] T.M. Apostol, Modular functions and Dirichlet series in number theory, Springer-Verlag, New York, 1990.

[2] S. Bochner, A new approach to almost periodicity, Proc. Nat. Acad. Sci., 48 (1962), 2039-2043.

[3] A.S. Besicovitch, Almost periodic functions, Dover, New York, 1954.

[4] C. Corduneanu, Almost Periodic Oscillations and Waves, Springer, New York, 2009.

[5] A.M. Fink, Almost Periodic Diflerential Equations, Lecture Notes in Math. Vol. 377, Springer-Verlag, New York, 1974.

[6] G.H. Hardy, E.M. Wright, An introduction to the theory of numbers, Oxford Science, Oxford, 1979.

[7] J.C. Lagarias, Mathematical quasicrystals and the problem of diffraction, Directions in Mathematical Quasicrystals, M. Baake and R. Moody, eds., CRM Monograph series, Vol. 13, AMS, Providence RI, 2000, 61-93.

[8] A.A. Karatsuba, S.M. Voronin, The Riemann zeta function. Walter de Gruyter \& Co., Berlin, 1992.

[9] J.M. Sepulcre, T. Vidal: Almost periodic functions in terms of Bohr's equivalence relation, Ramanujan J., 46 (1) (2018), 245-267; Corrigendum, ibid, 48 (3), 685-690 (2019).

[10] J.M. Sepulcre, T. Vidal: A generalization of Bohr's equivalence theorem, Complex Anal. Oper. Theory, 13 (4) (2019), 1975-1988.

[11] R. Spira, Sets of values of general Dirichlet series, Duke Math. J. 35 (1) (1968), 79-82. 
[12] C.E. Wilder, Expansion problems of ordinary linear differential equations with auxiliary conditions at more than two points, Trans. Am. Math. Soc., 18 (1917), 415-442. 